\title{
A Fast Method for In vitro Biomineralization of PVA/Alginate/Biphasic Calcium Phosphate Hydrogel
}

Lei Nie, Xingchen Li, Pengbo Chang, Shuang Liu, Qianqian Wei, Qingping Guo, Qiaoyun Wu, Lihong Fan, Okoro Oseweuba Valentine, and Amin Shavandi

\begin{abstract}
The development of biomineralized hydrogels with excellent cytocompatibility is of great importance for tissue engineering applications. Here, porous polyvinyl alcohol/alginate/biphasic calcium phosphate (BPS) hydrogels were fabricated via chemical and physical crosslinking methods, and the BPS hydrogels were in vitro biomineralized using urease in saturated calcium-phosphorus solution. For comparison, the BPS hydrogels were also treated using simulated body fluid (SBF) and Dulbecco's modified Eagle's medium with fetal bovine serum (DMEM-FBS), respectively. The physicochemical characterizations confirmed that bone-like apatite was quickly formed on the urease-biomineralized BPS hydrogels compared to the SBF and DMEM-FBS treated hydrogels. Additionally, the bone marrow derived mesenchymal stem cells (BMSCs), adhered and proliferated on the biomineralized hydrogels, were systematically analyzed using cell counting kit-8 (CCK-8). This paper demonstrated the potential urease for fast in vitro biomineralization on hydrogels to improve the cytocompatibility for bone tissue engineering applications.
\end{abstract}

Keywords: Hydrogels; Biomineralization; Cytocompatibility; Polymers.

\section{Introduction}

Bone tissue is composed of minerals in an organic matrix that serves as a reservoir of ions to maintain phosphocalcic equilibrium [1]. Scaffolds have been widely used to mimic the properties of bone tissue extracellular matrix (ECM) for the implantation of bone grafts to aid tissue regeneration [2,3]. 
Biomineralization is considered an effective approach to produce biomimetic scaffolds with improved osteogenesis capability. Biomineralized scaffolds could be fabricated via several in vitro methods, such as bioinspired [4], simulated body fluid (SBF) [5], and alternate-soaking treatment [6,7]. Our previous results have confirmed that Dulbecco's modified Eagle's medium with fetal bovine serum (DMEMFBS) treatment could facilitate the formation of bone-like apatite on polyvinyl alcohol/biphasic calcium phosphate (PVA/BCP) hydrogels [8]. However, the formation of bone-like minerals on polymer-based scaffolds remains a challenge as the polymer functional groups, including phosphate, phosphonate, carboxylate, and sulfonate, are implicated in regulating bone mineralization via hydrogen bonding and covalent attachment [9]. To circumvent this challenge, in this study, we fabricated hydrogel based on PVA, alginate, and BCP nanoparticles with hydroxy, carboxyl, and phosphate groups, then the hydrogels were biomineralized using urease, SBF, and DMEM-FBS, respectively, to obtain a fast biomineralization approach (Scheme 1). The formation of bone-like minerals on hydrogels was investigated by Fourier Transform Infrared Spectroscopy (FTIR), X-ray Diffraction (XRD), and Scanning Electron Microscope (SEM) images. Furthermore, the cytocompatibility of the biomineralized hydrogels was analyzed by culturing with the bone marrow derived mesenchymal stem cells (BMSCs).

\section{Experimental}

\subsection{Fabrication of PVA/Sodium Alginate/Biphasic Calcium Phosphate (BPS) Hydrogel}

Biphasic calcium phosphate (BCP) nanoparticles were synthesized via an aqueous precipitation reaction according to our previous reports $[10,12]$. Polyvinyl alcohol (PVA) solution $(8 \mathrm{wt} \%)$ as solution A, sodium alginate (SA) solution (4 wt $\%$ ) as solution B, and BCP slurry (50 wt\%), were prepared. Solution A was added into solution B and stirred for $48 \mathrm{~h}$ at $50{ }^{\circ} \mathrm{C}, \mathrm{BCP}$ slurry was poured 
into the above composite solution and stirred for $48 \mathrm{~h}$ at $60^{\circ} \mathrm{C}$. After that, the composite solution was poured into a 24-well plate, and the boric acid and calcium chloride $\left(\mathrm{CaCl}_{2}\right)$ were added as crosslinkers The samples were freeze-thawed for four cycles (frozen at $-20^{\circ} \mathrm{C}$ for $20 \mathrm{~h}$, thawed at room temperature for $4 \mathrm{~h}$ ). Finally, the samples were lyophilized at $-65{ }^{\circ} \mathrm{C}$ for $72 \mathrm{~h}$ to obtain BPS hydrogels. The designation of 40BPS, 50BPS, and 60BPS represents the final weight ratios of BCP nanoparticles of $40 \%, 50 \%$ and $60 \%$, respectively, and the mass ratio of SA to PVA was 1:2 for all samples.

\subsection{In vitro Biomineralization of BPS Hydrogel}

The saturated calcium phosphorous (SCP) solution was prepared by adding urea (30 g), dibasic sodium phosphate $\left(\mathrm{Na}_{2} \mathrm{HPO}_{4}, 3.5 \mathrm{~g}\right), \mathrm{CaCl}_{2}(6.9 \mathrm{~g})$ and sodium chloride $(\mathrm{NaCl}, 10.6 \mathrm{~g})$ into $250 \mathrm{~mL}$ of Millipore water. The prepared BPS hydrogels were immersed into the SCP solution, and urease was added and stirred at $37{ }^{\circ} \mathrm{C}$ to obtain biomineralized BPS (U-BioBPS) hydrogels. The BPS hydrogels were treated using SBF and DMEM-FBS for comparison, and the treated hydrogels were designated as S-BioBPS and F-BioBPS, respectively.

\subsection{Characterizations}

Fourier-transform infrared spectroscopy (FT-IR, PerkinElmer Spectrum 2), rheometer (TA, DHR, USA), X-ray diffraction (XRD, Rigaku Smartlab $9 \mathrm{~kW}$ ), cold field emission scanning electron microscope (SEM) and energy dispersive X-ray spectroscopy (EDX) were used to evaluate the characteristics of BPS and biomineralized BPS hydrogels. In addition, Cell Counting Kit-8 (CCK-8) analysis was employed to study the cytocompatibility of BPS and biomineralized BPS hydrogels after culturing with bone marrow derived mesenchymal stem cells (BMSCs, ATCC ${ }^{\circledR}$ PCS-500-012 ${ }^{\mathrm{TM}}$ ).

For more details about chemicals, composites preparation, biomineralization treatments, and characterization, please see the Supporting Information (SI). All of the data were reported as mean \pm 
$\operatorname{SD}(n=5)$.

\section{Results and discussion}

In this study, the reinforced porous BPS hydrogels with physical and chemical-crosslinking were fabricated. Based on the functional groups of the polymer chains such as hydroxy and carboxyl groups and calcium and phosphate ions in hydrogels, SBF, DMEM-FBS, and urease biomineralization approaches were used (Scheme 1). The needle-like BCP nanoparticles were obtained (Fig. 1A). The chemical interaction between PVA, alginate and BCP nanoparticles was analyzed using FT-IR (Fig. 1B). The peaks at 1033 and $599 \mathrm{~cm}^{-1}$ corresponded to the tension vibration and deformation vibration of $\mathrm{PO}_{4}{ }^{3-}$ for BCP nanoparticles. For BPS hydrogels, the appearance of peaks at 1028 and $1646 \mathrm{~cm}^{-1}$, which due to intense $\mathrm{C}-\mathrm{O}$ bands and carbonyl stretch, indicating the interaction between $\mathrm{BCP}$ nanoparticles, alginate and PVA, and the peak at $3357 \mathrm{~cm}^{-1}$ (O-H stretching) confirmed the crosslink between PVA and boric acid (Fig. S1) [11]. The peaks at 1646 and $1432 \mathrm{~cm}^{-1}$ contributed to -COOstretching vibration from alginate, indicating the "egg-box" structure of alginate in BPS hydrogels $[12,13]$. The BPS hydrogels displayed a porous interconnected structure using SEM observation, and such structure was not apparently influenced by BCP nanoparticles concentration in the range of 40 60 $\mathrm{wt} \%$ (Fig. S2). The stiffness of hydrogels was observed to be influenced by variations in BCP content, as illustrated by changes in the storage moduli $\left(\mathrm{G}^{\prime}\right)$. To ensure the framework of hydrogels for in vitro biomineralization, sample 40BPS was used for subsequent treatments (Fig. 1C).

Next, SBF, DMEM-FBS, and urease were used for in vitro biomineralization of the BPS hydrogels. FT-IR analysis (Fig. 2A) compares the biomineralized hydrogel sample to an un-biomineralized hydrogel sample, the peak at $870 \mathrm{~cm}^{-1}$ from biomineralized hydrogels was shown to be enhanced, with the observation attributed to the curved vibration of $\mathrm{CO}_{3}{ }^{2-}$ from the newly formed hydroxyapatite-like 
minerals. The peak at $1028 \mathrm{~cm}^{-1}$ was depressed due to the dissolution of BCP nanoparticles during the biomineralization process. Interestingly, the urease-biomineralized sample displayed a strong peak at $1385 \mathrm{~cm}^{-1}$, indicating the incorporation of carbonate ions [14]. Compared with SBF treatment, the carbonate apatite, known as bone-like apatite, was formed on DMEM-FBS and urease treated hydrogels, confirmed by XRD analysis (Fig. 2B). In addition, fukalite ((Ca4 $\left.\mathrm{Si}_{2} \mathrm{O}_{6}\left(\mathrm{CO}_{3}\right)(\mathrm{OH})_{2}\right), \mathrm{ICCD}$, 29-0308) was formed on urease-treated hydrogels. The introduction of silicate provides mineralization sites on the surface of urease-treated hydrogels to form silicic acid minerals [15]. However, it was observed that as the treatment hours increased, the formation of fukalite decreased while the content of bone-like apatite increased (Fig. S3). Based on the SEM images (Fig. 2C-H), more minerals were formed on BPS hydrogels by DMEM-FBS and urease biomineralization compared to when the SBF approach was employed. The broccoli flower-like minerals were observed on urease-biomineralized hydrogels, and the higher calcium ions were incorporated to form carbonate apatite, confirmed by EDX analysis (Fig. 2G-J).

Lastly, the cytocompatibility of biomineralized BPS hydrogels was evaluated using CCK-8 analysis (Fig. 3A). The results showed that the number of BMSCs on hydrogel increased over days, and there was a higher number of cells in urease-treated hydrogels than the hydrogels treated with FBS and DMEM-FBS. Although mineralization using SBF is a common and inexpensive method, however, mineral formation is slow, it may need at least one week. Similarly, the DMEM-FBS method could also promote the formation of bone-like apatite, but it is expensive, and the mineralization is slower than the SBF [8]. The urease-treatment approach could easily destroy the scaffold structure while treating the scaffold over two days and further decrease its mechanical property. However, such an approach could facilitate the formation of calcium-rich amorphous calcium phosphate, and then the 
bone-like minerals could be formed under urea and urease circumstances, and the minerals would cover the surface of hydrogels in $6 \mathrm{~h}$ [8] (Fig. S4). Meanwhile, the cytocompatibility of ureasetreatmented samples was significantly improved (Fig. 3B).

\section{Conclusion}

In summary, the porous BPS hydrogels were successfully fabricated, then SBF, DMEM-FBS, and urease were used for biomineralization on hydrogels. Comparing to SBF and DMEM-FBS approaches, urease treatment provides an effective strategy for the faster formation of bone-like apatite on hydrogels. Besides, the cytocompatibility of biomineralized hydrogels was greatly improved. The summary of three biomineralization approaches on BPS hydrogels provides valuable suggestions on selecting the suitable method for biomineralization for bone tissue engineering applications.

\section{Declaration of Competing Interest}

The authors declare no conflict of interest.

\section{Acknowledgments}

This research was supported by the Nanhu Scholars Program for Young Scholars of XYNU. Lei Nie acknowledges the support from Milky-T. Yang Yuan.

\section{References}

1. Y. Bala, D. Farlay, G. Boivin, Osteoporos Int. 24 (2013) 2153-2166.

2. D. Chen, C.X. Zhang, H.J. Huo, C.C. Ji, M. Sun, L. Nie, Mater. Lett. 229 (2018) 138-141.

3. L. Nie, C. Wang, R.X. Hou, X.Y. Li, M. Sun, J.P. Suo, Z. Wang, R.H. Cai, B.W. Yin, L. Fang, X.Y. Wei, H.Y. Yuan, SN Appl. Sci. 1(6) (2019).

4. K. Hadagalli, A.K. Panda, S. Mandal, B. Basu, ACS Appl. Bio Mater. 2 (2019) 2171-2184.

5. C. Zhang, M. Cao, J. Lan, P. Wei, Q. Cai, X. Yang, J. Biomed. Mater. Res. A. 104 (2016) 1968-1980.

6. Z.X. Meng, H.F. Li, Z.Z. Sun, W. Zheng, Y.F. Zheng, Mat. Sc. Eng. C. Mater. 33 (2013) 699-706.

7. J. Kong, B. Wei, T. Groth, Z. Chen, L. Li, D. He, R. Huang, J. Chu, M. Zhao, J. Biomed. Mater. Res. A. 106 (2018) 2714-2725.

8. L. Nie, X. Li, Z. Wang, K. Hu, R. Cai, P. Li, Y. Han, M. Sun, H. Yuan, J. Suo, S. Yang, Bioinspir. Biomim. Nan. 9 (2019) 122-128. 
9. K. Kepa, R. Coleman, L. Grøndahl, Biosurface and Biotribology. 1 (2015) 214-227.

10. L. Nie, Y. Deng, P. Li, R. Hou, A. Shavandi, S. Yang, ACS Omega. 5 (2020) 10948-10957.

11. L. Nie, Q. Wu, H. Long, K. Hu, P. Li, C. Wang, M. Sun, J. Dong, X. Wei, J. Suo, D. Hua, S. Liu, H. Yuan, S. Yang, J. Biomat. Sci. Polym. E. 30 (2019) 1636-1657.

12. L. Nie, Y. Deng, Y. Zhang, Q. Zhou, Q. Shi, S. Zhong, Y. Sun, Z. Yang, M. Sun, C. Politis, A. Shavandi, J. Appl. Polym. Sci. 138 (2021) 50433.

13. L. Nie, D. Chen, J. Suo, P. Zou, S. Feng, Q. Yang, S. Yang, S. Ye, Colloid. Surface. B. 100 (2012) 169-176.

14. T.E.L. Douglas, A. Łapa, S.K. Samal, H.A. Declercq, D. Schaubroeck, A.C. Mendes, P.V. der Voort, A. Dokupil, A. Plis, K.D. Schamphelaere, I.S. Chronakis, E. Pamuła, A.G. Skirtach, J. Tissue Eng. Regen. M. 11 (2017) 3556-3566.

15. R. B, C. J, S. H, C. F, I. F, L. H, Acta Biomater. Odontol. Scand. 2 (2016) 68-78.

A
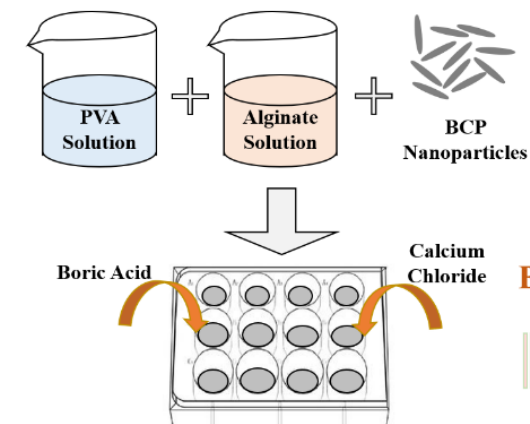

Freeze-thawing Freeze-dried
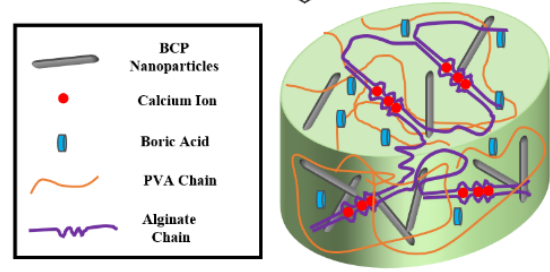

B

Method 1

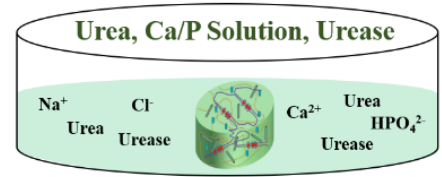

Biomineralization
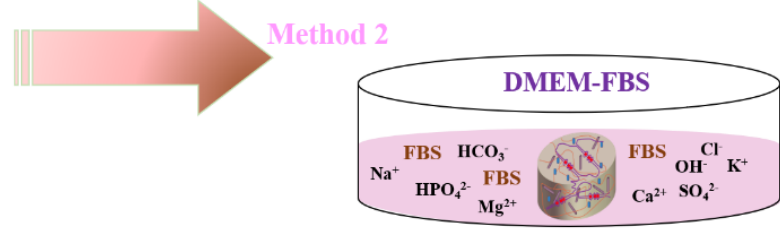

Method 3

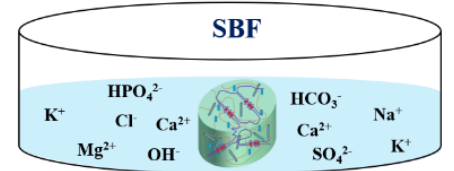

Scheme 1. (A) A schematic illustration of the preparation of PVA/sodium alginate/biphasic Calcium Phosphate (BPS) Hydrogel. (B) BPS hydrogels were treated via different methods for further biomineralization.
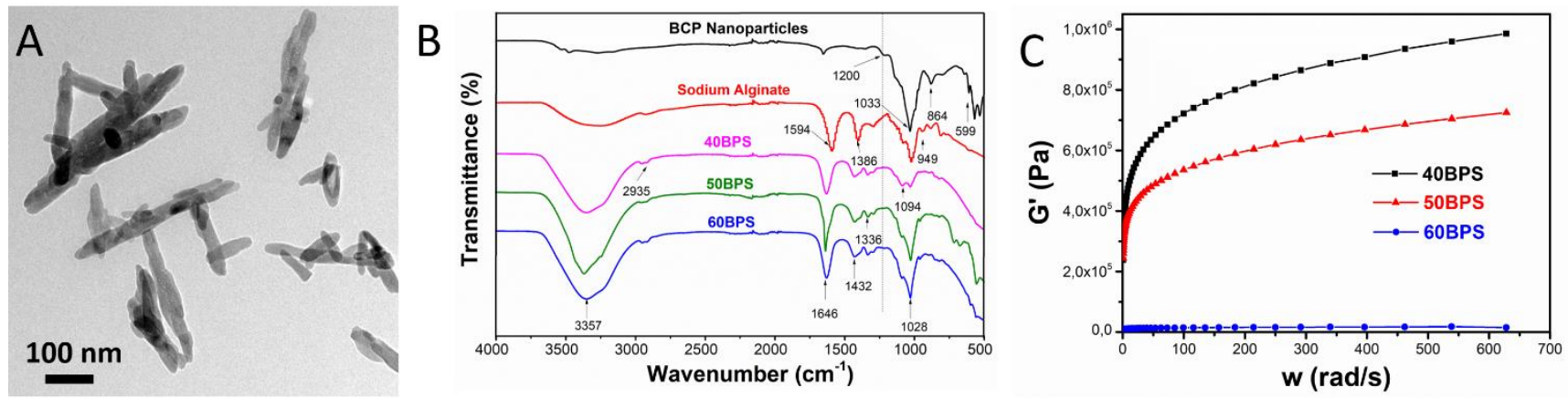

Fig. 1 (A) TEM image of BCP nanoparticles; (B) FT-IR spectrum of BCP nanoparticles, sodium alginate, and BPS hydrogels; (C) the change of storage modulus (G') of BPS hydrogels in term of frequency. 

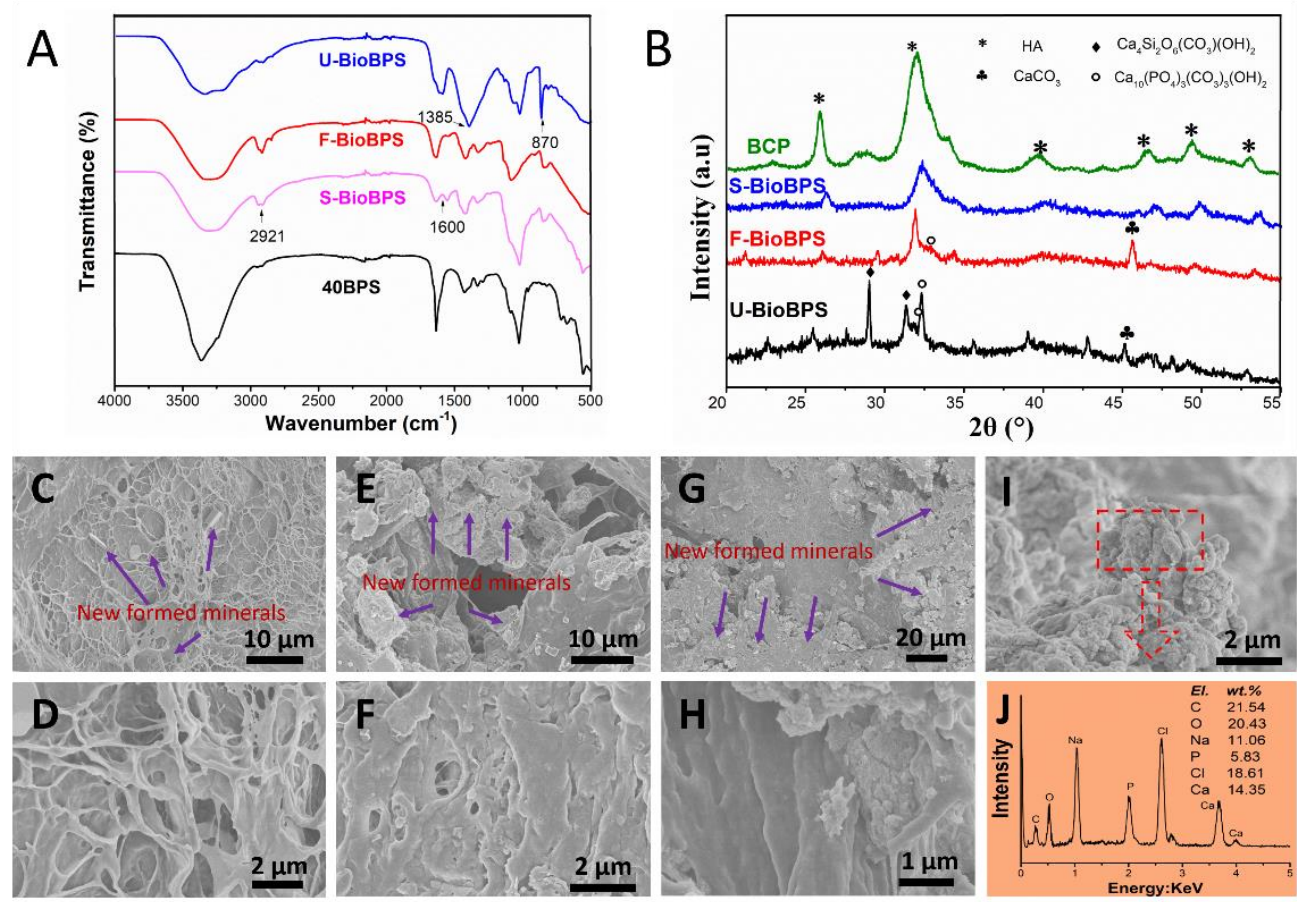

Fig. 2 (A) FT-IR spectrum of 40BPS hydrogel and biomineralized BPS hydrogels (U-BioBPS, F-BioBPS, and S-BioBPS, BPS hydrogels were biomineralized for $3 \mathrm{~h}$ for each approach); (B) XRD spectrum of BCP nanoparticles and biomineralized BPS hydrogels; SEM images of S-BioBPS (C, D), F-BioBPS (E, F), and U-BioBPS (G, H, I) hydrogels at different magnifications; (J) EDX analysis of UBioBPS hydrogel.
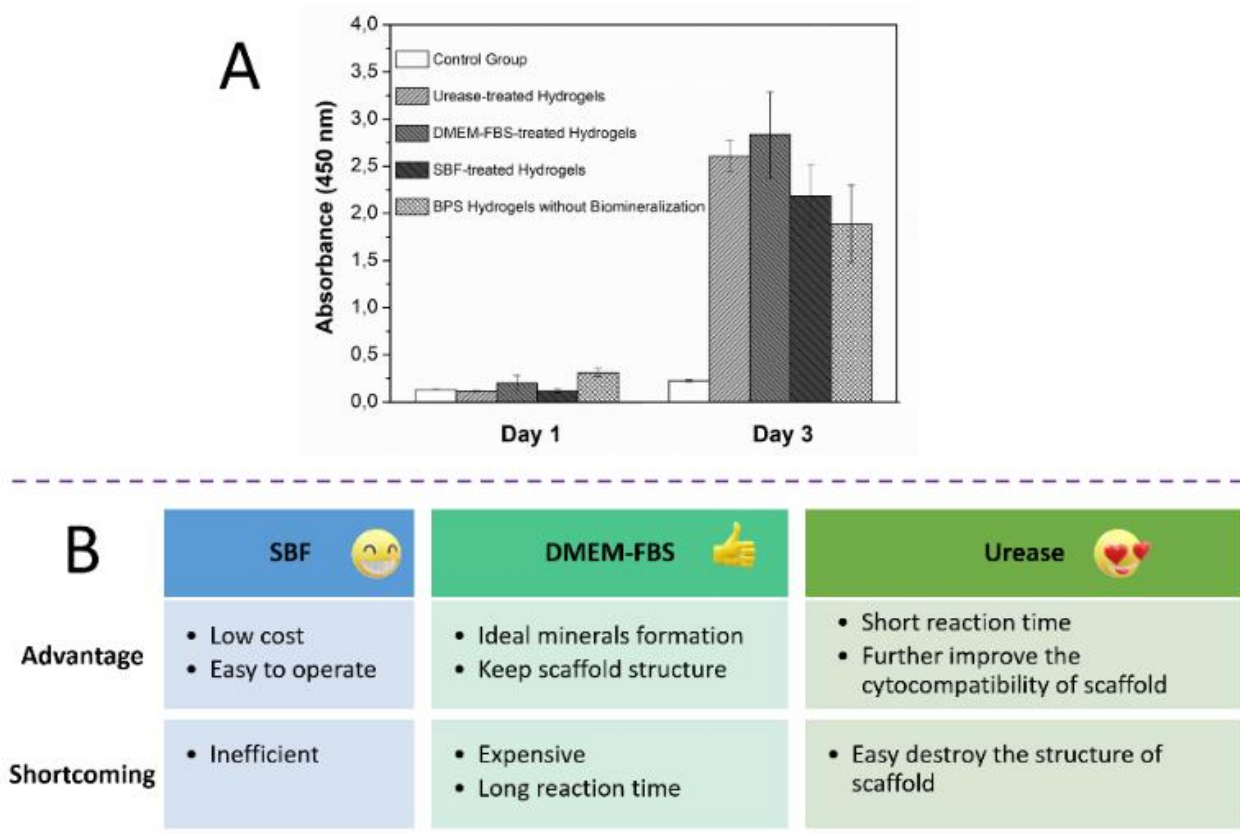

Fig. 3 (A) The cytocompatibility of BPS hydrogels and biomineralized BPS hydrogels were analyzed by CCK-8 after cultured with BMSCs for different days; (B) the advantage and shortcomings of different in vitro biomineralization approaches on scaffolds were summarized. 